\title{
Frequency stabilization of an ultraviolet semiconductor disk laser
}

\author{
David Pabœuf,* Peter J. Schlosser, and Jennifer E. Hastie \\ Institute of Photonics, SUPA, University of Strathclyde, Wolfson Centre, 106 Rottenrow, Glasgow G4 ONW, UK \\ *Corresponding author: david.paboeuf@strath.ac.uk
}

Received March 7, 2013; revised April 12, 2013; accepted April 13, 2013;

posted April 18, 2013 (Doc. ID 186639); published May 15, 2013

\begin{abstract}
We report a tunable, narrow-linewidth UV laser based on intracavity second-harmonic generation in a red semiconductor disk laser. Single-frequency operation is demonstrated with a total UV output power of $26 \mathrm{~mW}$. By servo-locking the fundamental frequency to a reference Fabry-Pérot cavity, the linewidth of the UV beam has been reduced to $16 \mathrm{kHz}$ on short timescales and $50 \mathrm{kHz}$ on a $1 \mathrm{~s} \mathrm{timescale,} \mathrm{relative} \mathrm{to} \mathrm{the} \mathrm{reference.} \mathrm{(C)} 2013$ Optical Society of America

OCIS codes: (140.7270) Vertical emitting lasers; (190.2620) Harmonic generation and mixing; (140.3610) Lasers, ultraviolet; (140.3425) Laser stabilization.

http://dx.doi.org/10.1364/OL.38.001736
\end{abstract}

During the last decade, optically pumped semiconductor disk lasers (SDLs) have become an attractive means to achieve tunable, high power, and near-diffraction-limited cw laser sources. The combination of bandgap engineering and efficient intracavity nonlinear conversion has enabled almost complete spectral coverage from the ultraviolet (UV) to the near IR [1-3]. Moreover, singlefrequency operation is readily achieved in a simple standing wave cavity, without spatial hole burning effects, due to the use of resonant periodic gain [4], and the external cavity accommodates mode-filtering elements such as birefringent filters (BRFs) or etalons, which enable single-frequency emission together with relatively broad tuning. Most of the demonstrations of single-frequency SDLs have been done in the near IR [4-6], but the visible has also been reached either directly [7] or via nonlinear conversion [ $\underline{8}-10]$ ], including commercial SDLs with linewidths in the megahertz range [11]. Achieving high spectral purity under normal laboratory conditions usually requires the use of active frequency stabilization. Holm et al. demonstrated a linewidth of $3 \mathrm{kHz}$ at $870 \mathrm{~nm}$ over $2 \mathrm{~s} \mathrm{[4]}$ and Rösener et al. a linewidth of $9 \mathrm{kHz}$ at $2 \mu \mathrm{m}$ over a short sampling time of $100 \mu \mathrm{s}$ [5] by locking their respective SDLs to a Fabry-Pérot (FP) reference cavity. Narrow linewidths, $<100 \mathrm{kHz}$, can also be achieved in free-running operation via careful engineering and isolation of the external cavity [12].

Here we describe the realization of a single-frequency UV-emitting laser based on intracavity second-harmonic generation in an AlGaInP-based SDL. Reduction of the laser linewidth is obtained by servo-locking the fundamental frequency to a reference FP cavity [13]. Compared with other frequency-stabilized UV laser systems that require successive external enhancement cavities, each with separate stabilization $[\underline{9}, 10,14]$, we benefit from a simpler setup due to the short fundamental wavelength and high finesse cavity that allow us to reach the UV with a single intracavity doubling step. High-spectralpurity UV emission opens new applications for SDLs such as interference lithography, UV spectroscopy [10], and metrology.

The experimental setup is described in Fig. 1 . The SDL gain structure contains an AlGaAs distributed Bragg reflector on top of which are 20 GaInP quantum wells (QWs) within a resonant subcavity for fundamental emission around $675 \mathrm{~nm}$, with the QWs being distributed evenly between the 10 available field antinodes. The structure is very similar to the one reported previously in [3]. It was inserted as an end mirror in a four-mirror cavity including two mirrors $\left(\mathrm{M}_{1}\right.$ and $\left.\mathrm{M}_{2}\right)$ with radii of curvature of $100 \mathrm{~mm}$ and the end mirror $\left(\mathrm{M}_{3}\right)$ having a radius of curvature of $25 \mathrm{~mm}$. A $500-\mu \mathrm{m}$-thick single crystal diamond heatspreader with low birefringence was bonded onto the intracavity surface of the SDL structure for efficient thermal management [15]. The gain medium was then clamped into a water-cooled brass mount, with the water temperature set to $0^{\circ} \mathrm{C}$. It was optically pumped by a Coherent Inc. Verdi laser with up to $4 \mathrm{~W}$ at $532 \mathrm{~nm}$. The losses due to reflection by the heatspreader were $20 \%$, so that the actual maximum pump power incident at the SDL was $3.2 \mathrm{~W}$. The intracavity frequency doubling of the red beam was achieved with a 7-mm-thick betabarium borate (BBO) crystal cut for type I phase matching at $674 \mathrm{~nm}\left(\varphi=35.2^{\circ}\right)$, inserted at the beam waist position between $\mathrm{M}_{2}$ and $\mathrm{M}_{3}$. The crystal was held in an aluminium mount with indium foil, and the end facets were antireflection coated for both red $(R<0.1 \%)$ and UV $(R<0.2 \%)$ wavelengths. All cavity mirrors were coated to have high reflectivity $(R>99.9 \%)$ for the red wavelengths, with $\mathrm{M}_{2}$ and $\mathrm{M}_{3}$ highly transmitting in the

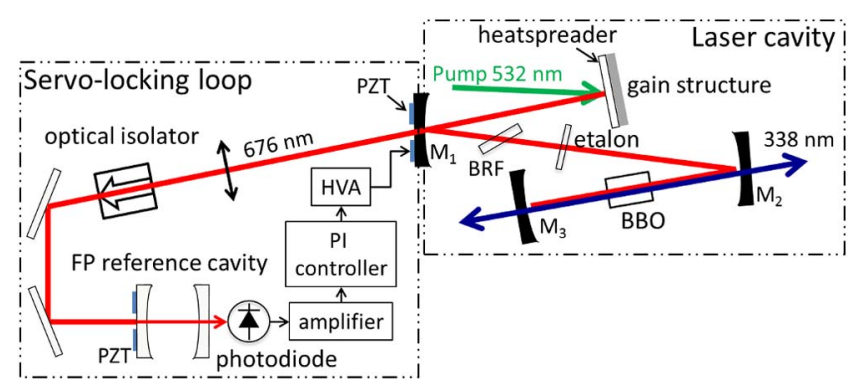

Fig. 1. Schematic of the single-frequency UV emitting laser cavity and of the servo-locking loop. $\mathrm{M}_{1}, \mathrm{M}_{2}, \mathrm{M}_{3}$, cavity mirrors; $\mathrm{BRF}$, birefringent filter; $\mathrm{BBO}$, beta-barium borate crystal; PZT, piezoelectric transducer; FP, Fabry-Pérot; PI, proportional integrator; HVA, high voltage amplifier. 
UV ( $T \sim 98 \%)$ so that two UV beams with equal powers exited the cavity. These beams are sometimes recombined using a cavity mirror with high reflectivity at the second harmonic to reflect one beam back through the crystal; however, for the single-frequency beam with a coherence length much longer than the laser cavity, interference effects would be detrimental. The distances between the SDL and $\mathrm{M}_{1}, \mathrm{M}_{1}$ and $\mathrm{M}_{2}$, and $\mathrm{M}_{2}$ and $\mathrm{M}_{3}$ were, respectively, 72,310 , and $93 \mathrm{~mm}$. The beam waist diameters at the gain structure were calculated as $80 \mu \mathrm{m}$ in the tangential plane and $87 \mu \mathrm{m}$ in the sagittal, and in the BBO crystal, as 71 and $80 \mu \mathrm{m}$, respectively.

In order to achieve single-frequency emission, a 2-mmthick quartz plate at Brewster's angle, acting as a BRF, and a $1 \mathrm{~mm}$ thick uncoated FP glass etalon (Suprasil 311) were inserted in the cavity. The diamond heatspreader, being uncoated, also behaved as an etalon and contributed to the mode filtering. The single-frequency behavior was monitored by coupling a leak of the fundamental beam through $\mathrm{M}_{1}$ into a confocal FP cavity with a $750 \mathrm{MHz}$ free spectral range and finesse of 10. Figure 2(a) shows the resulting spectrum obtained for an absorbed pump power of $3.2 \mathrm{~W}$ and a total UV output power of $26 \mathrm{~mW}$, clearly demonstrating single-frequency operation. The nonlinear output coupling was $\sim 0.1 \%$. By rotating the $\mathrm{BRF}$, tuning of the fundamental wavelength was possible, which resulted in a tuning of the UV wavelength; however, as the heatspreader is a fixed etalon, tuning is not continuous in the current setup. Single-frequency operation was regained by slightly adjusting the tilt of the glass etalon and changing the orientation of the BBO crystal to optimize the phase matching, and hence the UV output power, at the new wavelength. Continuous tuning may be achieved in a future setup via the use of a wedged, antireflection-coated heatspreader [16]. An overall single frequency tuning range of $5 \mathrm{~nm}$ in the UV was achieved [Fig. 2(b)]. Maximum output power was obtained for a fundamental wavelength of $676.4 \mathrm{~nm}(338.2 \mathrm{~nm}$ in the UV).

In order to isolate the laser cavity from outside perturbations, the optical table was floated and the SDL was enclosed in a box and mounted on a breadboard, which
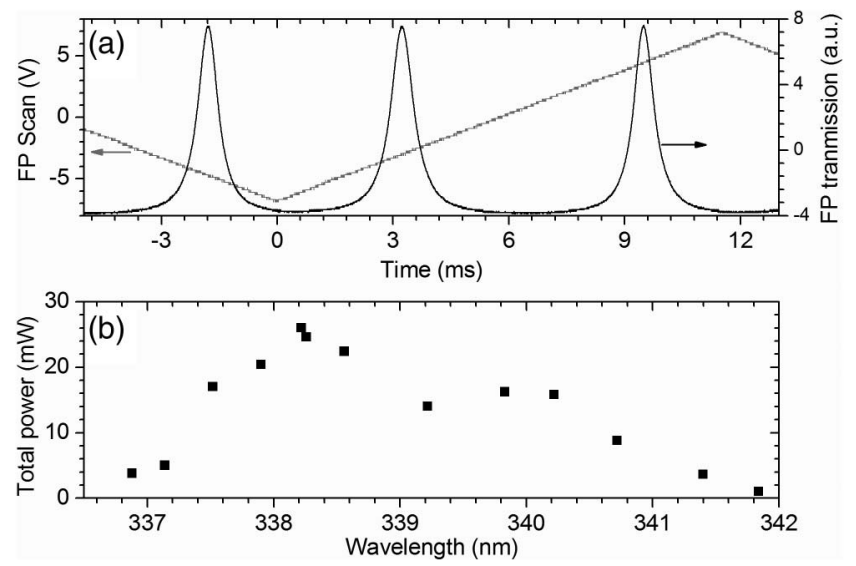

Fig. 2. (a) Transmission of the scanned FP cavity showing single-frequency operation for a total UV output power of $26 \mathrm{~mW}$ and (b) total single frequency UV output power measured as the $\mathrm{BRF}$ is rotated to tune the fundamental wavelength. was insulated from the optical table by $25.4 \mathrm{~mm}$ thick Sorbothane feet at each corner. To evaluate the freerunning frequency variations of this source, we used the FP cavity as a frequency reference. An optical isolator was used to prevent perturbing feedback into the laser. The length of the FP cavity was set with a piezoelectric transducer (PZT) so that the laser frequency fluctuated on the side of the cavity resonance peak. The transmitted signal was then recorded for $1 \mathrm{~s}$. Due to the rather large frequency fluctuations, this measurement can only give an estimation of the laser linewidth. The free-running linewidth was estimated to be $\sim 3 \mathrm{MHz}$ for the red beam, which resulted in a linewidth around $6 \mathrm{MHz}$ for the UV laser beam. Computing the Fourier transform of the signal leads to the frequency noise power spectral density (PSD) shown in Fig. 3, indicating that the bandwidth of frequency fluctuations is limited to a few kilohertz, which is, as for most solid-state lasers, several orders of magnitude lower than that of a typical dye laser or laser diode.

To stabilize the frequency, we used the so-called "side of fringe" technique [13] using the setup shown in Fig. 1. The signal detected at the output of the reference FP cavity was amplified and sent to a commercial proportional integrator servo-controller (Precision Photonics model LB1005). The locking reference was set at half the maximum of the FP cavity fringe. After amplification by a high-voltage amplifier (PI model E-503), the correction signal was applied to a PZT (PI model P-010.00H) carrying cavity mirror $\mathrm{M}_{1}$.

With this arrangement, the laser frequency could be locked for several minutes, the limiting factor being the drift of the reference cavity, which induced mode hops of the laser or multimode behavior. From the residual error signal, one can compute the laser frequency noise PSD relative to the reference cavity. Figure 3 shows the resulting PSD measured over $1 \mathrm{~s}$ when the laser was locked. At the time of the locking experiment, the total UV output power was $20 \mathrm{~mW}$. One can clearly see that the frequency noise was significantly reduced as compared to the free-running case over the full noise bandwidth. At low frequencies the noise level is reduced by a factor of $10^{6}$. With the exception of some localized peaks between $200 \mathrm{~Hz}$ and $1 \mathrm{kHz}$, the PSD is almost flat, with a noise level around $10^{5} \mathrm{~Hz}^{2} / \mathrm{Hz}$. From the integration of the PSD, a standard deviation of $25 \mathrm{kHz}$ is deduced for the fundamental frequency.

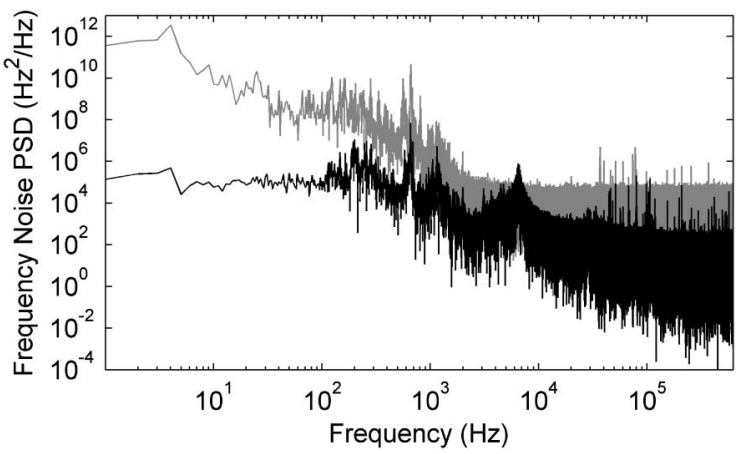

Fig. 3. Frequency noise power spectral densities (PSDs) of the red laser beam measured over a $1 \mathrm{~s}$ long sampling time for the free-running laser (gray curve) and the locked laser (black curve). 


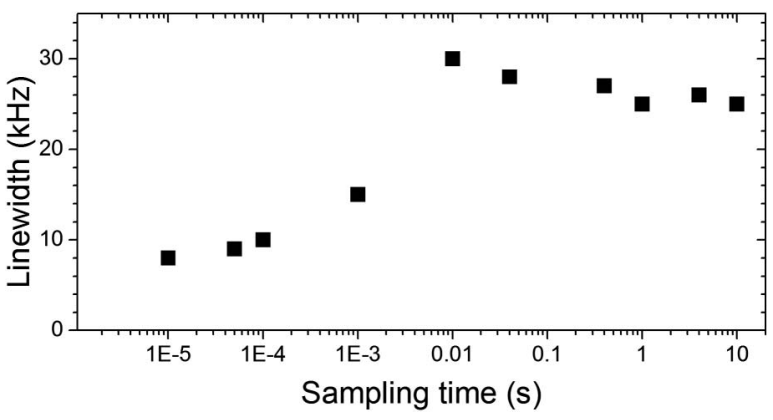

Fig. 4. Fundamental laser linewidth relative to the FP cavity measured over different sampling times. $P_{\text {totaluv }}=20 \mathrm{~mW}$.

A closer look at the PSD shows that more than half of the overall noise level is contained in the $100 \mathrm{~Hz}-1 \mathrm{kHz}$ range. We suspect that these noise components originate from mechanical resonances in the laser cavity. The relative increase of the noise level for frequencies above $100 \mathrm{~Hz}$ is related to the correction bandwidth of our servo-locking system, which is currently limited by the PZT to only $250 \mathrm{~Hz}$. We expect that by reducing the weight of the cavity mirror, currently a standard $6 \mathrm{~mm}$ thick optic, the correction bandwidth will be increased, thus further decreasing the laser linewidth. The broad peak around $6 \mathrm{kHz}$ is due to an electronic oscillation of the servo loop. The gain of the loop must be carefully set to minimize this oscillation while maintaining an efficient noise correction.

Figure 4 shows the fundamental laser linewidth measured over different time scales ranging from $10 \mu \mathrm{s}$ to $10 \mathrm{~s}$. The laser linewidth remains between 25 and $30 \mathrm{kHz}$ over long sampling times and steeply decreases below $10 \mathrm{~ms}$ to reach a value of $8 \mathrm{kHz}$ at $10 \mu \mathrm{s}$. This decrease of the linewidth when measured on short timescales is to be expected with noise dominated by low frequencies [17]. Moreover, the fact that the linewidth peaks around $10 \mathrm{~ms}$ and remains nearly constant over longer timescales confirms, as observed with the PSD, the majority of the frequency noise is contained within a $1 \mathrm{kHz}$ bandwidth.

In conclusion, we have demonstrated a tunable, narrow-linewidth UV laser based on intracavity frequency doubling of a single-frequency red SDL. By stabilizing the frequency to a reference FP cavity, a linewidth as narrow as $16 \mathrm{kHz}$ was achieved for the UV beam. Over a $1 \mathrm{~s}$ long sampling time the relative linewidth of the UV beam has been measured as $50 \mathrm{kHz}$. To the best of our knowledge, this is the narrowest linewidth achieved from an SDL with nonlinear frequency conversion. Further improvements of both the UV output power and the linewidth are expected via optimization of the nonlinear output coupling and increasing the bandwidth of the servo-locking system to lock the frequency to a higher finesse cavity, respectively. We also aim to achieve continuous tuning by using a wedged heatspreader.

This work is supported by the UK Engineering and Physical Sciences Research Council (EPSRC) under grant EP/I022791/1. The SDL gain structure was grown by Dr. A. B. Krysa at the EPSRC National Centre for III-V Semiconductors, University of Sheffield, UK.

\section{References}

1. S. Calvez, J. E. Hastie, M. Guina, O. G. Okhotnikov, and M. D. Dawson, Laser Photon. Rev. 3, 407 (2009).

2. N. Schulz, J.-M. Hopkins, M. Rattunde, F. Bussières, and J. Wagner, Laser Photon. Rev. 2, 160 (2008).

3. J. E. Hastie, L. G. Morton, A. J. Kemp, M. D. Dawson, A. B. Krysa, and J. S. Roberts, Appl. Phys. Lett. 89, 061114 (2006).

4. M. A. Holm, D. Burns, A. I. Ferguson, and M. D. Dawson, IEEE Photon. Technol. Lett. 11, 1551 (1999).

5. B. Rösener, S. Kaspar, M. Rattunde, T. Töpper, C. Manz, K. Köhler, O. Ambacher, and J. Wagner, Opt. Lett. 36, 3587 (2011).

6. A. Rantamäki, A. Chamorovskiy, J. Lyytikäinen, and O. G. Okhotnikov, IEEE Photon. Technol. Lett. 24, 1378 (2012).

7. L. G. Morton, H. D. Foreman, J. E. Hastie, M. D. Dawson, and E. Riis, in Advanced Solid-State Photonics (Optical Society of America, 2007), paper WB7.

8. M. Jacquemet, M. Domenech, G. Lucas-Leclin, P. Georges, J. Dion, M. Strassner, I. Sagnes, and A. Garnache, Appl. Phys. B 86, 503 (2007).

9. Y. Kaneda, J. M. Yarborough, L. Li, N. Peyghambarian, L. Fan, C. Hessenius, M. Fallahi, J. Hader, J. V. Moloney, Y. Honda, M. Nishioka, Y. Shimizu, K. Miyazono, H. Shimatani, M. Yoshimura, Y. Mori, Y. Kitaoka, and T. Sasaki, Opt. Lett. 33, 1705 (2008).

10. J. Paul, Y. Kaneda, T. Wang, C. Lytle, J. V. Moloney, and R. J. Jones, Opt. Lett. 36, 61 (2011).

11. Coherent Inc. Press Release No. 1277 (2011).

12. A. Laurain, M. Myara, G. Beaudoin, I. Sagnes, and A. Garnache, Opt. Express 18, 14627 (2010).

13. R. L. Barger, M. S. Sorem, and J. L. Hall, Appl. Phys. Lett 22, 573 (1973).

14. S. Vasilyev, A. Nevsky, I. Ernsting, M. Hansen, J. Shen, and S. Schiller, Appl. Phys. B 103, 27 (2011).

15. A. J. Kemp, G. J. Valentine, J.-M. Hopkins, J. E. Hastie, S. A. Smith, S. Calvez, M. D. Dawson, and D. Burns, IEEE J. Quantum Electron. 41, 148 (2005).

16. A. J. Maclean, A. J. Kemp, S. Calvez, J.-Y. Kim, T. Kim, M. D. Dawson, and D. Burns, IEEE J. Quantum Electron. 44, 216 (2008).

17. S. Kaspar, M. Rattunde, T. Töpper, C. Manz, K. Köhler, and J. Wagner, Appl. Phys. Lett. 100, 031109 (2012). 